\title{
JÓVENES, ESCUELA TÉCNICA Y TRABAJO EXPERIENCIAS DE ESCOLARIZACIÓN EN CONTEXTOS DE POBREZA URBANA (ROSARIO, SANTA FE)
}

\author{
Florencia Debonis * \\ Universidad Nacional de Rosario, CONICET, Argentina \\ flodebonis@hotmail.com \\ Marilín López Fittipaldi ${ }^{\text {* }}$ \\ Universidad Nacional de Rosario, CONICET, Argentina \\ marilinlopez@gmail.com \\ Mercedes Saccone ${ }^{* *}$ \\ Universidad Nacional de Rosario, CONICET, Argentina \\ sacconemercedes@gmail.com
}

Recibido: 7/08/2020 - Aceptado: 14/10/2020

\section{Resumen}

En este escrito recuperamos el trabajo realizado en dos proyectos de investigación colectivos, ambos inscritos en una línea de investigación centrada en procesos socioeducativos en contextos de pobreza y distintas violencias en la ciudad de Rosario (Santa Fe, Argentina) y orientados desde un enfoque socio-antropológico (Achilli, 2005).

Analizamos la relación entre escuela técnica y trabajo en las experiencias de jóvenes que viven en contextos de pobreza urbana: los sentidos sobre la escolarización y su vinculación con el trabajo desde la perspectiva de los y las jóvenes; los sentidos sobre la modalidad técnico profesional; el sostenimiento de la escolaridad en articulación con sus condiciones de vida.

Sostenemos que los/as jóvenes llegan a esta escuela por diversos motivos, no necesariamente vinculados a la elección de la modalidad técnico profesional en administración y gestión, no obstante, el conocimiento y la valoración sobre la misma se va construyendo a lo largo de la experiencia escolar con sentidos heterogéneos. Estos sentidos están atravesados fuertemente por el vínculo que se establece entre la educación y el trabajo en términos de posibilitar el acceso a mejores condiciones de vida.

\footnotetext{
*Doctoranda en Antropología en la Facultad de Filosofía y Letras, Universidad de Buenos Aires (FFyL, UBA). Becaria Doctoral del Consejo Nacional de Investigaciones Científicas y Técnicas (CONICET). Licenciada en Antropología, Facultad de Humanidades y Artes, Universidad Nacional de Rosario (FHyA, UNR). Adscripta en la Escuela de Antropología, (FHyA, UNR). Miembro del Programa de Antropología y Educación del Centro de Estudios Antropológicos en Contextos Urbanos (CEACU, FHyA, UNR).

${ }^{*}$. Doctoranda en Antropología en la Facultad de Filosofía y Letras, Universidad de Buenos Aires (FFyL, UBA). Becaria Doctoral del Consejo Nacional de Investigaciones Científicas y Técnicas (CONICET). Licenciada en Antropología, Facultad de Humanidades y Artes, Universidad Nacional de Rosario (FHyA, UNR). Docente auxiliar de 1era categoría en la Escuela de Antropología (FHyA, UNR). Miembro del Programa de Antropología y Educación del Centro de Estudios Antropológicos en Contextos Urbanos (CEACU, FHyA, UNR).

*** Doctora en Antropología, Facultad de Filosofía y Letras, Universidad de Buenos Aires (FFyL, UBA). Becaria Postdoctoral del Consejo Nacional de Investigaciones Científicas y Técnicas (CONICET) en la Unidad Ejecutora en Ciencias Sociales Regionales y Humanidades, Consejo Nacional de Investigaciones Científicas y Técnicas - Universidad Nacional de Jujuy (UE-CISOR, CONICET-UNJu). Licenciada y Profesora en Antropología, Facultad de Humanidades y Artes, Universidad Nacional de Rosario (FHyA, UNR). Miembro del Programa de Antropología y Educación del Centro de Estudios Antropológicos en Contextos Urbanos (CEACU, FHyA, UNR).
} 
Palabras clave: Jóvenes - Escuela técnica - Trabajo - Experiencias de escolarización - Pobreza urbana.

\author{
YOUTH, TECHNICAL SCHOOL AND WORK \\ SCHOOLING EXPERIENCES IN URBAN POVERTY CONTEXTS \\ (ROSARIO, SANTA FE)
}

\begin{abstract}
In this paper we retrieve the work carried out under two collective research projects, both enrolled in a research line focused on socio-educational processes in contexts of poverty and various forms of violence in Rosario city (Santa Fe, Argentina) and driven by a socio-anthropological approach (Achilli, 2005).

We analyze the relationship between technical school and work in the experiences of young people living in contexts of urban poverty: the meanings of schooling and its connection with work from the perspective of young people; the senses about professional technical modality; the sustaining of schooling in articulation with their living conditions. We maintain that young people arrive at this school for various reasons, not necessarily linked to the choice of the technical-professional modality in administration and management, however, knowledge and assessment of it is built throughout the school experience with heterogeneous senses. These senses are strongly crossed by the link established between education and work, in terms of enabling access to better living conditions.
\end{abstract}

Keywords: Youth - Technical school - Work - Schooling experiences - Urban poverty.

\title{
Introducción
}

En este escrito analizamos la relación entre escuela técnica y trabajo en las experiencias de jóvenes que viven en contextos de pobreza urbana ${ }^{1}$ Recuperamos el trabajo realizado en el marco de dos proyectos de investigación colectivos, focalizados en los sentidos sobre la escolarización que construyen jóvenes en el tránsito de experiencias socioeducativas permeadas por procesos de desigualdad social y, articuladamente, en los sentidos del trabajo que construyen las y los jóvenes que asisten a una escuela secundaria técnica en la ciudad de Rosario ${ }^{2}$. Ambos se inscriben en una línea de investigación centrada en procesos socioeducativos en contextos de pobreza y distintas violencias en la ciudad de Rosario (provincia de Santa Fe, Argentina), articulada al Programa de Antropología y Educación del Centro de Estudios Antropológicos en Contextos Urbanos (Facultad de Humanidades y Artes, Universidad Nacional de Rosario).

El proceso de investigación se llevó adelante desde un enfoque socio-antropológico que recupera la tradición etnográfica de la Antropología desde una perspectiva crítica (Achilli, 2005). El trabajo de campo, para el cual recurrimos a distintas estrategias de construcción de la información -talleres, entrevistas grupales, observaciones y cuestionarios-, se desarrolló en una escuela secundaria técnica con orientación en administración y gestión ubicada en el Distrito Oeste de la ciudad. A esta escuela asisten jóvenes que habitan en barrios y villas cercanas, muchos/as de los/as cuales trabajan en condiciones precarias y tienen niños/as a cargo de su cuidado, condiciones que atraviesan el desenvolvimiento de sus experiencias escolares.

Específicamente nos interesa abordar: 1) los sentidos sobre la escolarización y su vinculación con el trabajo desde la perspectiva de los y las jóvenes; 2) los sentidos sobre la modalidad técnico profesional; 3) el sostenimiento de la escolaridad en articulación con las condiciones de vida, en particular, las posibilidades de compatibilizar los tiempos escolares y laborales.

A partir del análisis realizado, sostenemos que los y las jóvenes llegan a esta escuela por diversos motivos, que no necesariamente están vinculados a la elección de la modalidad técnico profesional en administración y gestión, no obstante, el conocimiento y la valoración sobre la misma se va construyendo a lo largo de la experiencia escolar con sentidos heterogéneos. Estos sentidos están atravesados fuertemente por el vínculo que se establece entre la educación y el trabajo en términos de posibilitar el acceso a mejores condiciones de vida.

\footnotetext{
${ }^{1}$ Este trabajo reelabora parte de los avances del proceso de investigación plasmados en Nemcovsky et. al. (2020). 2Proyecto: "Jóvenes y experiencias socioeducativas. Un estudio antropológico de los sentidos sobre la escolarización en contextos de pobreza urbana" (PID SeCyT UNR 1HUM 520, 2016-2019) y Proyecto: "Jóvenes y Educación Técnica. Un estudio antropológico de los sentidos acerca del trabajo en la escuela técnica" (AEI-FONIETP NRU 212, 2017-2018). Ambos bajo la dirección de Mariana Nemcovsky y la co-dirección de Gabriela Bernardi, y en los cuales también participaron Marina Santos, Mirna Calamari, Noelia Martínez, Mara Dobry y Eliana Maiolino.
} 
REVISTA DE LA ESCUELA DE CIENCIAS DE LA EdUCACIÓN, AÑO 17, NRO. 16, VOL. 2, JULIO A DICIEMBRE DE 2021. PÁGINAS 47-56. ISSN 2362-3349 (EN LíNEA). JÓVENES, ESCUELA TÉCNICA Y TRABAJO EXPERIENCIAS DE ESCOLARIZACIÓN EN CONTEXTOS DE POBREZA URBANA (ROSARIO, SANTA FE). FLORENCIA DEBONIS. MARILÍN LÓPEZ FITTIPALDI. MERCEDES SACCONE.

\section{Jóvenes, escuela técnica y trabajo. Aspectos teórico-metodológicos de la investigación}

En este apartado presentamos algunas precisiones referidas al enfoque socio-antropológico relacional (Achilli, 2005) desde el cual se orientó el proceso de investigación ${ }^{3}$.

Desde esta perspectiva se parte de entender la realidad como totalidad concreta (Kosik, 1967), abordando la problemática de estudio a partir de las relaciones entre distintas dimensiones que la configuran, reconociendo el carácter conflictivo, contradictorio y en permanente movimiento de los procesos sociales (Achilli, 2005, p. 17). Este enfoque privilegia el conocimiento de la cotidianeidad social recuperando las prácticas y sentidos que generan los sujetos en sus relaciones e interacciones. Retomando a Heller (1987), quien destaca el carácter heterogéneo e histórico de la vida cotidiana, ésta es entendida como configuración de "un conjunto de prácticas, relaciones, significaciones diversas y heterogéneas que construyen sujetos particulares al interior de una realidad concreta" (Achilli, 2005, p. 22). La cotidianeidad social es, a la vez, "mediadora hacia lo no cotidiano" (Heller, 1987, p. 25) y allí puede encontrarse "la evidencia más sólida de los procesos estructurales", tanto como "intersticios" que abren alternativas en la configuración de los procesos sociales (Rockwell, 2018, p. 239).

Partimos de considerar que la juventud es una construcción social, histórica, cultural y relacional que responde a la vez a una franja epocal y a enclasamientos sociales (Bourdieu, 2002), por lo tanto, los y las jóvenes viven su cotidianeidad en el tránsito de experiencias diferenciadas según su clase social, su lugar de residencia y la generación a la que pertenecen. Retomando a E.P. Thompson (1963), entendemos a las experiencias en tanto huellas que las condiciones concretas de existencia dejan en la conciencia social. Se trata de un proceso activo por el cual un conjunto de elementos socio-culturales -prácticas, sentidos, saberes, valores- son apropiados por los sujetos, en nuestro caso los/as jóvenes, en los entornos inmediatos por los que transitan cotidianamente, entre ellos el escolar. Apropiación que se produce en el seno de "las relaciones constituidas entre los diferentes actores significativos que intervienen en una situación o proceso determinado" (Menéndez, 2010, p.26). Es decir, un proceso educativo, en sentido amplio, un "campo de conformación sociocultural de prácticas y significaciones humanas", de "'enseñanzas' y 'aprendizajes' presentes en distintos ámbitos, además del específicamente escolar", por lo tanto, un "campo constitutivo de matrices socioculturales que incluyen prácticas, conjuntos de valores, modos de relaciones subjetivas, ideologías, etc." (Achilli, 1999, p.6). Desde esta perspectiva, la escuela es una "institución" que "existe como un 'concreto real', donde el Estado se encuentra presente pero no de manera absoluta, es decir, no determina totalmente las interacciones entre los sujetos involucrados ni el sentido de las prácticas" (Ezpeleta y Rockwell, 1985, p.74). Cada escuela se concibe entonces, como producto de una permanente construcción social y por ello, subrayan Ezpeleta y Rockwell (1985), la necesidad de observarla en su cotidianeidad.

En las últimas décadas ha sido extenso el desarrollo de investigaciones sobre trayectorias educativas de jóvenes en contextos de pobreza urbana, que las caracterizan como fragmentadas (Foglino, Falconi y López, 2008; Guzmán y Saucedo, 2007; Kessler, 2002; Terigi, 2010; Tiramonti, 2012; otros), intentando recuperar su diversidad y contraponiendo las "trayectorias escolares teóricas" o "ideales" a las "reales". Predomina el análisis de los sentidos de la escolarización para los y las jóvenes. La mayoría de los estudios revisados (Llinás, 2009; Montesinos, Sinisi y Schoo, 2009; Weiss, 2012) coincide en que continúa siendo valorada positivamente por los/as jóvenes y sus familias, aunque son diversos los sentidos que se le atribuyen. Además de la importancia como medio para un trabajo formal o "ser alguien en la vida", la escuela es significada como "espacio de vida juvenil" (Maldonado, 2000; Molina, 2013; Weiss, 2012).

Respecto a la educación secundaria técnica en particular, sobresalen estudios -algunos de corte historiográfico- centrados en el análisis de las políticas y características de la modalidad, los cuales suelen prestar especial atención al vínculo entre educación y trabajo (y/o modelo económico/productivo y político) y a las características, en términos generales, que adquieren las instituciones y el subsistema en distintos momentos socio-históricos (Almandoz, 2010; Binstock y Cerrutti, 2017; Dussel y Pineau, 1995; Gallart, 2006; otros). Ciertas investigaciones de carácter extensivo analizan las trayectorias de estudiantes y las inserciones laborales y educativas de egresados en la educación técnica de nivel medio (Álvarez, 2013; Jacinto, 2018; entre otros) ${ }^{4}$. Aunque escasas, algunas investigaciones analizan lo que sucede a nivel de los cotidianos escolares y las experiencias de los sujetos, atendiendo a la vinculación entre educación y trabajo en las trayectorias de jóvenes en la educación técnica (específicamente de mujeres, Bloj, 2017; Seoane, 2013), los sentidos sobre la "formación para el trabajo", y/o los sentidos sobre el trabajo que construyen jóvenes que asisten a escuelas secundarias técnicas en distintos contextos provinciales (Butti y Florez, 2018; Cruz, Cortéz, Moreno y Pereyra, 2019).

A nivel local la temática ha sido incipientemente explorada, aun cuando la provincia de Santa Fe es una de las jurisdicciones con mayor cantidad de instituciones de ETP de Argentina (INET, 2017). En ese contexto, resultan relevantes aquellos trabajos realizados desde el CEACU (FHyA, UNR), que indagan sobre experiencias escolares de jóvenes en la educación secundaria técnica (Saccone, 2020); sobre políticas destinadas a la educación técnica de nivel medio provincial (Debonis, López Fittipaldi y Saccone, 2018; Saccone, 2019), así como los avances en la línea de indagación que venimos desarrollando acerca de los sentidos sobre trabajo que construyen jóvenes que viven en contextos de pobreza y asisten a una

\footnotetext{
${ }^{3}$ Para una descripción detallada de la perspectiva teórico-metodológica ver Nemcovsky et. al. (2020).

${ }^{4}$ Existe también una línea de investigación sobre la "transición" y/o relación entre educación y trabajo de jóvenes que asisten o egresaron de escuelas secundarias (no técnicas) (Corica, 2012; Filmus, Miranda y Zelarayan, 2001; Kornblit, 2004; Llomovatte, 1991; Otero y Miranda, 2005; otros).
} 
REVISTA DE LA ESCUELA DE CIENCIAS DE LA EdUCACIÓN, AÑO 17, NRO. 16, VOL. 2, JULIO A DICIEMBRE DE 2021. PÁGINAS 47-56. ISSN 2362-3349 (EN LíNEA). JÓVENES, ESCUELA TÉCNICA Y TRABAJO EXPERIENCIAS DE ESCOLARIZACIÓN EN CONTEXTOS DE POBREZA URBANA (ROSARIO, SANTA FE). FLORENCIA DEBONIS. MARILÍN LÓPEZ FITTIPALDI. MERCEDES SACCONE.

escuela secundaria técnica (Nemcovsky et al., 2020). De este modo, el presente trabajo supone una continuación de esa línea de indagación, que apunta a profundizar el conocimiento acerca de las experiencias escolares de jóvenes en la ETP en la ciudad de Rosario.

El trabajo de campo se llevó a cabo durante los años 2016 y 2017, como hemos dicho, en una escuela secundaria pública de gestión estatal, de Modalidad Técnico Profesional con orientación en Administración y Gestión que cuenta con tres turnos (matutino, tarde y noche), ubicada en el Distrito Oeste. Recurrimos a distintas estrategias de construcción de la información que nos permitieron documentar en profundidad sentidos y prácticas que construyen los y las jóvenes: observaciones, entrevistas y distintas estrategias grupales, que se complementaron con algunos recursos de carácter exploratorio, como la implementación de cuestionarios. Focalizamos en el turno noche, ya que aquellos/as jóvenes que trabajan se inscriben en mayor proporción en ese turno 5 .

\section{Los sentidos sobre la escolarización y su vinculación con el trabajo desde la perspectiva de los y las jóvenes}

Para describir los sentidos que los y las jóvenes construyen en torno a la escolarización, en primer lugar, retomaremos los motivos expresados por éstos/as acerca de cómo llegan a esta escuela. De acuerdo a la orientación en administración y gestión, la escuela posee un plan de estudios con preeminencia de materias del área contable y contenidos de las llamadas "ciencias empresariales". No obstante, como pudimos documentar, los/as estudiantes manifiestan diversas motivaciones que no necesariamente están vinculadas con la elección de dicha modalidad. Sobresalen los argumentos que refieren a: la cercanía del hogar al establecimiento, "porque me queda más cerca" (R9, 19/06/17, T.N. 1er año), relacionado, a su vez, con las dificultades para afrontar los gastos de transporte ${ }^{6}$,"[antes] gastaba mucho en colectivo" (R10, 15/08/17, T.N. 5to año), y las facilidades que tal cercanía supone para la organización del grupo doméstico, "es más fácil para mi hermana que ella trabaja cuestión de que me pueda retirar" (R9, 19/06/17, T.N. 1er año); el conocimiento previo de la institución, a partir de la experiencia personal o a través de familiares y amigos/as, "Elegí porque ya vienen mis hermanos [sic]" (R9, 19/06/17, T.N. 1er año); el deseo más general de finalizar la escuela secundaria "y tener mi título secundario" (R10, 15/08/17, T.N. 4to año) y/o "tener un mejor trabajo" (R9, 19/06/17, T.N. 1er año).

Como señalan otros estudios (Cerletti, 2009), en estos procesos de elección de escuelas se ponen en juego definiciones y opciones que activamente construyen los sujetos, pero dentro de condiciones que le son dadas. De este modo, vemos que las motivaciones señaladas se encuentran constreñidas por las condiciones materiales de vida de los/as jóvenes y sus familias y por la limitada oferta de instituciones educativas del nivel en la zona en que habitan. De hecho, el Distrito Oeste es uno de los distritos de la ciudad con menor número de establecimientos educativos y con menor capacidad escolar por habitante en edad teórica de cursar los niveles obligatorios (Instituto de Gestión de Ciudades, 2010). A su vez, la escasa matrícula con que cuenta la escuela favorece la incorporación de estudiantes que fueron expulsados o rechazados en otras instituciones ${ }^{7}$. Según los y las jóvenes que "repitieron" algún año más de una vez en otras escuelas, esta institución es la "única que los acepta": "Vine a esta escuela porque es la única escuela que me aceptaron porque venía repitiendo seguido en otras escuelas [sic]" (R9, 19/06/17, T.N. 1er año).

A su vez, las características de esta escuela -la posibilidad de cursar bajo la "Nocturnidad" que elimina el cursado en doble turno, la relación con profesores/as y preceptores/as, entre otras- favorecen el sostenimiento de la escolaridad, sobre todo para los/as estudiantes que trabajan. Así los y las jóvenes expresan: "Bueno yo lo deje el ante años pasado bueno por eso llegue acá pero me gusta porque los compañeros son muy buenos y los profe te comprenden porque yo trabajo y a veces llegue un poco tarde pero me entienden [sic]" (R10, 09/08/17, T.N. 3er año).

En tal sentido, es posible evidenciar, como señala Cerletti (2009), las "diversas y complejas articulaciones entre las dimensiones de la agencia humana y las limitaciones estructurales propias de cada contexto socio-histórico" (p.311) que configuran los procesos de selección de la escuela.

1.1 Sentidos que los y las jóvenes construyen sobre los procesos de escolarización

A continuación, describiremos los sentidos que construyen, en el transcurrir de sus experiencias, con respecto a la escolarización en términos amplios y, posteriormente, profundizaremos en aquellos que refieren a la modalidad técnico profesional. La experiencia es un "proceso activo", a través del cual los/as jóvenes van apropiándose de un conjunto de elementos socio-culturales diversos. Así, el paso por la escuela va imprimiendo huellas que pueden dar lugar a procesos de construcción y resignificación de los

\footnotetext{
${ }^{5}$ En el turno noche la escuela ofrece una "opción pedagógica y didáctica" de cursado mediado por "materiales didácticos y por las tecnologías de la información y la comunicación", denominada "Nocturnidad", que suprime el doble turno (ver Saccone, 2019).

${ }^{6}$ A diferencia de otras localidades que tienen boleto estudiantil gratuito, en la ciudad de Rosario, la tarifa del transporte urbano de pasajeros para estudiantes sólo cuenta con una bonificación del $50 \%$ respecto de la tarifa básica (medio boleto estudiantil).

${ }^{7}$ En el turno noche, la escuela posee dos divisiones de 1er año (con un promedio de 40 inscriptos cada una), número que disminuye marcadamente en los años subsiguientes (llegando a contar con una división con menos de 10 estudiantes en 6 to año). A su vez, dentro de cada curso, la matrícula desciende considerablemente a medida que transcurre el ciclo lectivo.
} 
REVISTA DE LA ESCUELA DE CIENCIAS DE LA EdUCACIÓN, AÑO 17, NRO. 16, VOL. 2, JULIO A DICIEMBRE DE 2021. PÁGINAS 47-56. ISSN 2362-3349 (EN LínEA). JÓVENES, ESCUELA TÉCNICA Y TRABAJO EXPERIENCIAS DE ESCOLARIZACIÓN EN CONTEXTOS DE POBREZA URBANA (ROSARIO, SANTA FE). FLORENCIA DEBONIS. MARILÍN LÓPEZ FITTIPALDI. MERCEDES SACCONE.

sentidos sobre la escolaridad "dentro de determinados límites", "bajo condiciones que vienen dadas" (Thompson, 1981, p.19).

En términos amplios, los y las estudiantes construyen sentidos diferenciales sobre la escolarización. Expresan valoraciones acerca de la escuela como espacio privilegiado para el aprendizaje y en relación con proyecciones de futuro (ya sea para continuar estudios posteriores o conseguir trabajo); pero también refieren asistir a la escuela por "obligación".

Sobresale una valoración positiva de la escuela en tanto posibilitaría incorporar conocimientos para "no ser un burro" (R1, 12/10/16, T.M. 1er y 2do año), como distinción frente a aquellos/as que no concurren y concibiendo a la escuela como espacio privilegiado para el aprendizaje. La valorización de los aprendizajes se vincula a la idea de "ser alguien": "retomé este año porque sin la secundaria no sos nada" (R7, 22/06/17, T.N. 3er año).

Esta valoración de la escuela también aparece ligada a proyecciones de futuro, por su importancia para conseguir trabajo, mejorar las inserciones laborales que se tienen y/o para continuar estudios superiores. Sin embargo, en relación al trabajo, más que un énfasis en los conocimientos adquiridos, se destaca la relevancia que se le adjudica a la obtención del título, del "diploma para conseguir trabajo" (R10, 15/08/17, T.N. 4to año). Esta valoración del título se desprende, en parte, de las propias experiencias de búsqueda laboral de las y los jóvenes que en repetidas veces se presentan a entrevistas "pero no te llaman" (R12, 24/08/17, T.N. 4to y 6 to año).

Ligado a los proyectos de futuro, los sentidos sobre la escolarización también giran en torno a la continuidad de los estudios en el nivel superior. Algunos/as estudiantes afirman que desean continuar estudios vinculados a la terminalidad de la escuela en la "facultad de ciencias económicas, contabilidad" o "administración de empresas" (R8, 22/06/17, T.N. 5to año). Otros/as se inclinan hacia carreras que no se vinculan necesariamente con la orientación, como el magisterio, "para maestra jardinera" o "de grado" (R12, 24/08/17, T.N. 4to y 6 to año).

Por último, sobresale la "obligación" de ir a la escuela, que a veces se refuerza en el ámbito familiar: "algunos vienen sólo porque los padres los mandan", "no me gusta venir a la escuela. Pero me obligan" (R2, 12/10/16, T.M. 1er y 2do año). Cabe señalar, no obstante, que existen matices en cómo se expresa el sentido en torno a asistir a la escuela por "obligación", especialmente en relación con las edades de los y las estudiantes. De este modo, encontramos que se plantea más frecuentemente entre aquellos/as más jóvenes.

Finalmente, destacamos que las distintas construcciones de sentido sobre la escolarización se pueden ver tensionadas cuando las condiciones materiales de vida de los/as jóvenes y sus familias dificultan el sostenimiento de la asistencia a la escuela, por ejemplo, al no contar con los recursos económicos suficientes para afrontar el gasto del transporte, requisito mínimo para poder concurrir.

\section{Sentidos sobre la modalidad técnico profesional. Entre la apropiación y el desconocimiento}

2.1 Desconocimiento y confusiones respecto a la modalidad técnico profesional

En parte, los sentidos sobre la escuela que construyen los y las jóvenes se configuran a partir de cierto desconocimiento/confusiones sobre la modalidad técnica. En ello pueden influir distintos procesos.

En primer lugar, a diferencia de lo documentado por otras investigaciones que dan cuenta del lugar relevante que tiene la valoración positiva de la educación técnica a la hora de elegir la institución entre jóvenes de sectores populares (Maturo, 2018), los y las jóvenes llegan a esta escuela por otros motivos, que se imponen por sobre a elección de la modalidad. A la vez, notamos que tal desconocimiento/confusiones se produce entre estudiantes que se encuentran cursando. Aspectos que suelen atribuirse a la modalidad técnica, como la mayor cantidad de años que supone el cursado, los espacios curriculares denominados "talleres" y las "prácticas", están atravesados por tal desconocimiento y confusiones.

$\mathrm{E}:$ bueno, ahora tienen taller con esta profe.

Ao2: pero taller de qué.

Ao1: de Marketing.

Ao2: o sea ¿la técnica de qué sería?

E: en Administración.

Ao1: es como te ayuda a estar en una empresa (R15, 08/11/17, T.N. 3er año).

Estas confusiones se vinculan, en parte, al poco tiempo que llevan cursando las y los jóvenes en esta escuela, pudiendo tratarse de los primeros años o incorporaciones producidas en el transcurso del año escolar. También a las características y condiciones particulares de esta escuela, las cuales se fueron configurando históricamente en la trama de las políticas educativas y las transformaciones que estas propiciaron en la educación secundaria técnica. A partir de la Ley Federal de Educación № 24.195 (1993), la escuela, como muchas otras instituciones de educación media técnica, se convirtió en Polimodal en bienes y servicios. Con la sanción de la Ley de Educación Técnico Profesional № 26.058 (2005) y de la Ley de Educación Nacional № 26.206 (2006), se vuelve a reconocer a la Educación Técnico Profesional como una de las modalidades para el sistema educativo nacional. A nivel de las escuelas, el proceso de implementación de la modalidad se dio lentamente, por lo que recién en el año 2016 se gradúa la primera cohorte con el título secundario de "Técnico en Administración y Gestión". Incluso, ante la ausencia de 
REVISTA DE LA ESCUELA DE CIENCIAS DE LA EdUCACIÓN, AÑO 17, NRO. 16, VOL. 2, JULIO A DICIEMBRE DE 2021. PÁGINAS 47-56. ISSN 2362-3349 (EN LíNEA). JÓVENES, ESCUELA TÉCNICA Y TRABAJO EXPERIENCIAS DE ESCOLARIZACIÓN EN CONTEXTOS DE POBREZA URBANA (ROSARIO, SANTA FE). FLORENCIA DEBONIS. MARILÍN LÓPEZ FITTIPALDI. MERCEDES SACCONE.

cargos docentes suficientes, los talleres fueron impartiéndose parcial o tardíamente, profundizando las confusiones que se expresan entre los y las estudiantes.

Aa1: ...materias, nos suman materias, nos sacan materias

$\mathrm{E}:$ ¿qué materia les sumaron?

Aa2: eh... ¿la última cuál es? (...) Recursos Humanos.

E: ¿y cuándo empezaron? (...)

Aa2: hace un mes.

Aa1: un mes, menos (R4, 12/10/16, T.M. 3er, 4to y 5to año).

Además, a diferencia de lo que ocurre en secundarias técnicas con otras orientaciones, en esta escuela el trabajo en talleres con conocimientos específicos de la orientación en administración y gestión presenta muchas similitudes con lo que se trabaja en las clases de las demás materias, aportando mayor confusión.

La incorporación gradual de las "prácticas profesionalizantes" (PP) establecidas como obligatorias para el 6to año en el nivel secundario de ETP provincial (Decreto $N^{\circ} 1446 / 11,2011$ ), también plantea dificultades para su concreción que pueden contribuir a generar ciertas confusiones respecto a las mismas. A partir del trabajo de campo realizado, observamos que algunos/as jóvenes no están al tanto directamente de su existencia o del modo en que se están llevando adelante. A su vez, los/as docentes a cargo de las PP destacan dificultades para realizar las prácticas fuera del ámbito escolar (por trabas burocráticas en el establecimiento de acuerdos marco, porque las actividades asignadas a los/as jóvenes en las organizaciones/empresas no cumplirían con los objetivos pedagógicos establecidos), optando por recurrir a otras estrategias (cursos de formación en espacios laborales, creación de "empresas simuladas" en el ámbito escolar, otros)

A la vez, en el turno noche se cursa bajo la "Nocturnidad", que implicó, como mencionamos, la concentración del cursado presencial en un sólo turno (17.30 a 22.30 horas), suprimiendo el "doble turno" tradicionalmente asociado a la escolarización secundaria técnica, y completando el cursado presencial a través de una plataforma virtual. Sin embargo, ello se dificulta por las transformaciones que se produjeron en el Plan Conectar Igualdad luego del cambio de gobierno a nivel nacional en $2015^{8}$. También, por las condiciones materiales del establecimiento, que asumen un deterioro significativo. Producto de la historia de cada escuela permeada por procesos más generales, estas condiciones estructuran el ámbito en que trabajan y conviven docentes y estudiantes, a la vez que "obligan a ciertas prácticas, posibilitan ciertas relaciones y delimitan el horizonte de lo cotidiano en cada escuela" (Rockwell, 1987, p.36).

2.2 Sentidos sobre la modalidad técnico profesional y su relación con el trabajo

Entre los sentidos que relacionan la modalidad técnico profesional con el trabajo, se destacan aquellos configurados en torno a los aprendizajes escolares específicos de la orientación y en relación al título que la misma otorga.

En cuanto a los conocimientos específicos, hay jóvenes que consideran que éstos les resultan útiles y están vinculados a los trabajos que poseen actualmente. Plantean que los conocimientos escolares les sirven para las tareas que realizan, sobre todo quienes trabajan en la construcción, "porque tenés que hacer mucho cálculo con la matemática" (R14, 24/08/17, T.N. 3er año). Aunque tal relación se construye con los trabajos presentes -generalmente caracterizados por la precariedad, la informalidad y la inestabilidad- que no suelen ser los deseados por ellos/as.

Algunos/as jóvenes consideran que los aprendizajes específicos les facilitarían la inserción laboral o educativa futura en ámbitos vinculados con el área contable: "lo que aprendo en la escuela me sirve para trabajar de lo que yo quiero trabajar, digamos, no encuentro relación con lo que hago ahora (...) me gustaría trabajar en algo contable" (R14, 24/08/17, T.N. 3er año). Aunque también hay jóvenes que destacan su relevancia para inserciones laborales futuras que no necesariamente se vinculan al área en cuestión.
E: ¿y algún otro piensa que le puede llegar a servir?
Ao: y porque si llegás a primera [división del fútbol argentino], tenés que tener algún, algo que te haga plata, son fábricas y esas cosas, y después te ayudan.
E: claro, para saber administrarte.
Ao: claro, todo eso.
E: claro, para saber administrarte con los sponsors.
Ao: para saber también, porque nos enseñaron... (R13, 24/10/17, T.N. 5to año).

Podemos advertir que son múltiples las relaciones que los y las estudiantes establecen entre los aprendizajes específicos de la orientación y el trabajo, no obstante, otros/as jóvenes consideran que tal relación no existe. Dicho planteo aparece ante la disociación que encuentran entre los trabajos deseados y

${ }^{8}$ El Plan Conectar Igualdad se crea en 2010 como una política socio-educativa a nivel nacional a través de la distribución de una computadora portátil a cada estudiante y docente junto con lineamientos pedagógicos para su utilización. En diciembre de 2015, a partir del cambio de gobierno nacional, comienza a desmantelarse progresivamente, hasta su reemplazo por el Plan "Aprender Conectados" en 2018. 
REVISTA DE LA ESCUELA DE CIENCIAS DE LA EdUCACIÓN, AÑO 17, NRO. 16, VOL. 2, JULIO A DICIEMBRE DE 2021. PÁGINAS 47-56. ISSN 2362-3349 (EN LíNEA). JÓVENES, ESCUELA TÉCNICA Y TRABAJO EXPERIENCIAS DE ESCOLARIZACIÓN EN CONTEXTOS DE POBREZA URBANA (ROSARIO, SANTA FE). FLORENCIA DEBONIS. MARILÍN LÓPEZ FITTIPALDI. MERCEDES SACCONE.

la orientación de la escuela, "a mí no me gusta nada de economía, nada, nada, nada. Me gusta todo lo que es de la policía, gendarmería todo eso" (R12, 24/10/17, T.N. 6to año) ${ }^{9}$.

En cuanto al título secundario técnico, algunos/as jóvenes lo valoran positivamente en relación a la cantidad de años de duración que supone el plan de estudios, lo que se plantea como "mejor" que otras opciones (como la escuela secundaria orientada o la educación media para adultos) a la hora de conseguir trabajo. También es valorado positivamente en tanto certifica un cursado que supone una carga horaria mayor a partir del doble turno, argumentando que:

Ao: si vos vas a pedir trabajo yo creo que van a considerar más una persona que hace el doble turno, que está acostumbrada a ir todo el día en un lugar, a cumplir un horario, que a una persona que fue, qué sé yo, tres veces por semana dos horas, supongamos (R14, 24/08/17, T.N. 3er año).

Lo que en ambas perspectivas se pone en juego es la asociación del título técnico con la posibilidad de acreditar un mayor tiempo de permanencia en la escuela -ya sea en años u horas de cursada-, lo cual, desde la perspectiva de estos/as estudiantes, podría ser ponderado al momento de insertarse en el mundo del trabajo. No obstante, ello es tensionado por algunos/as estudiantes al contraponer otros aspectos, tales como la edad aproximada con la que finalizarían el nivel secundario en caso de continuar cursando en la educación secundaria técnica. No debemos olvidar que muchos/as de los y las jóvenes que concurren a esta escuela poseen experiencias escolares marcadas por interrupciones temporales de la escolaridad y/o la repetición de algún/os año/s de cursado. A su vez, esta tensión aparece asociada, por un lado, a ciertos trabajos/opciones formativas que no requieren específicamente de un título de secundaria en la modalidad y, por otro, a la proyección familiar.

Se trata, desde el análisis que estamos proponiendo, de complejos procesos de apropiación en los que los sujetos, en el curso de sus experiencias escolares y en el cruce con diversas experiencias vitales, logran resignificar los objetos culturales disponibles (Rockwell, 2018). De este modo, a escala de la cotidianeidad social, los títulos pueden cobrar sentidos diferentes, no anticipables, a partir de análisis realizados en otras escalas (cfr. Bourdieu y Passeron, 1979). Como señala Rockwell (2009), el énfasis dado a la reproducción como elemento constitutivo de toda práctica escolar, supone una abstracción cuyo peligro radica en "el ocultamiento de variaciones significativas como instancias de contradicción o de resistencia frente al proceso de reproducción dominante" (Rockwell, 2009, p.134). Podemos preguntarnos, entonces, si las construcciones de sentido que realizan los y las jóvenes en torno al título que esperan obtener no conllevan un cuestionamiento, así sea de modo fragmentario o incipiente, a la condición subordinada que ocuparían dentro de la estructura laboral existente.

Otra cuestión a considerar es que la apropiación de sentidos se produce en las relaciones que se entablan entre diferentes actores (Menéndez, 2010) y, por lo tanto, en las relaciones intra e intergeneracionales en que se articulan y constituyen las "biografías particulares" de los y las jóvenes (Sanchez, 2005). De este modo, vemos que, en parte, los sentidos que valoran positivamente el título de la modalidad como habilitador para conseguir un "mejor trabajo", se construyen en los intercambios con los pares y, fundamentalmente, con los/as adultos/as con quienes interactúan cotidianamente y que, al decir de Gramsci (2004), "cumplen siempre la educación de los jóvenes" en sentido amplio. Así, los y las jóvenes suelen remarcar la influencia que sus docentes tienen en algunas decisiones que toman respecto a la escuela y, especialmente, en la valoración positiva del título de la modalidad, "yo una vez me quise cambiar, el año pasado creo que era porque no quería hacer un año más hasta 6to y él [director] me habló, me dijo que me quede, me dijo que es mejor" (R12, 24/10/17, T.N. 6to año).

\section{Sostenimiento de la escolaridad y condiciones de vida. Posibilidades de compatibilizar los tiempos escolares y laborales}

En este apartado nos interesa profundizar en las dificultades con que se encuentran los y las jóvenes para compatibilizar los tiempos escolares y laborales, ya que es frecuente que quienes asisten a esta escuela, sobre todo al turno noche, estén trabajando y tengan a cargo tareas domésticas y de cuidado (Nemcovsky, et al., 2020). Así, destacan estas tareas como obstaculizadoras para el sostenimiento de la escolaridad, "al tener un bebé chiquito no podía estar mucho tiempo fuera de casa" (R10, 15/08/17, T.N. 5to año).

Ao: Yo estaba estudiando mecánica y dejé porque eran 2 turnos a la mañana y a la tarde, en la escuela (...) dejé en 3ro porque me cansé y empecé a ir a la feria a trabajar y me gustó la plata (R7, 22/06/17, T.N. 3er año).

Una estrategia que despliegan para poder compatibilizar los tiempos destinados a dichas tareas con los que requiere la escuela es el cambio de turno.

\footnotetext{
${ }^{9}$ Esta opción es considerada por los y las jóvenes como una salida laboral con cierta estabilidad y, a la vez, como respuesta a una necesidad, en relación a las situaciones de inseguridad que se viven en los barrios que habitan. Como hemos profundizado en otra arista de la investigación más amplia de la que se desprende este escrito (Nemcovsky et al., 2020), estos sentidos se ven tensionados frente al accionar de las fuerzas de seguridad en esos mismos contextos barriales, experimentado por las y los jóvenes como abusivo y violento.
} 
REVISTA DE LA ESCUELA DE CIENCIAS DE LA EdUCACIÓN, AÑO 17, NRO. 16, VOL. 2, JULIO A DICIEMBRE DE 2021. PÁGINAS 47-56. ISSN 2362-3349 (EN LínEA). JÓVENES, ESCUELA TÉCNICA Y TRABAJO EXPERIENCIAS DE ESCOLARIZACIÓN EN CONTEXTOS DE POBREZA URBANA (ROSARIO, SANTA FE). FLORENCIA DEBONIS. MARILÍN LÓPEZ FITTIPALDI. MERCEDES SACCONE.

Ao: en 1er año, bueno, yo trabajaba como ayudante de albañil entonces vine a la noche (...) me quedé sin trabajo, me volví a la mañana, hice 2 do y hasta 3er año, mitad de año, y ahora me volví a pasar a la noche (R14, 24/08/17, T.N, 3er año).

Estas idas y venidas entre turnos a partir de los cambios en la situación laboral, así como las llegadas tarde o la entrega de trabajos fuera de término, son toleradas por la escuela para evitar la interrupción de la escolaridad. Aun cuando dichas facilidades brindadas por la institución permiten el sostenimiento de la escolaridad, los trabajos a los que suelen acceder conllevan un desgaste físico, resultando en cansancio y esfuerzo para atender o participar de las clases.

Ao1: cansa... cansa el hecho de decir no podés llegar a tu casa y descansar.

Ao2: ¿me entiende? yo llegué hace un rato y vine para la escuela.

$\mathrm{E}:$ ¿y qué pensás en ese momento?, ¿qué decís?

Ao1: tengo sueño.

E: ¿y qué pensás que no te quedás a dormir ahí y decís "voy"?

Ao2: no porque... yo sé adónde quiero llegar y sé lo que tengo que pasar para llegar, cada uno llega adonde puede por su esfuerzo (R14, 24/08/17, T.N. 3er año).

Otro aspecto que dificulta el trabajo en clase es la asistencia con los/as hijos/as pequeños/as que requieren atención, al no contar con un espacio específico y con personal que pueda encargarse de su cuidado, obstaculizando el desenvolvimiento de la clase y, particularmente, los procesos de aprendizaje de las jóvenes madres.

Distintos estudios (Foglino, Falconi y López 2008; García, 2013; Sinisi, Montesinos y Schoo, 2010) han subrayado que el tiempo escolar está continuamente tensionado por el tiempo que demandan las inserciones laborales de quienes trabajan, el cuidado de los/as hijos/as, el embarazo, las tareas del hogar, entre otras. Sin embargo, como han señalado Sinisi, Montesinos y Schoo (2010), y también lo hemos documentado en nuestra investigación, "acercarse, retirarse, esperar, explorar otros caminos, revincularse, proyectar, son acciones que implican decisiones -no siempre conscientes ni racionales- que se configuran en la compleja zona de articulación entre agencia y estructura" (p.72), procesos que permiten complementar y complejizar la mirada de algunas indagaciones de corte cuantitativo (SITEAL, 2013) que analizan la problemática de la interrupción de los estudios. De este modo, sostenemos que, aun cuando las experiencias vitales de los y las jóvenes están atravesadas por procesos y condiciones similares, no siempre se expresan en recorridos escolares semejantes, evidenciando las múltiples y diversas apropiaciones que realizan los sujetos.

\section{Reflexiones finales}

A lo largo del escrito profundizamos en el análisis de la relación entre escuela y trabajo desde las experiencias de jóvenes que asisten a una escuela técnica y viven en contextos de pobreza urbana.

Identificamos que manifiestan diversos motivos por los cuales asisten, no necesariamente vinculados a la elección de la modalidad técnico profesional en administración y gestión. Se trata de opciones constreñidas por las condiciones materiales de vida y por la limitada oferta de instituciones educativas del nivel en la zona en que habitan. Sin embargo, los y las estudiantes van apropiándose de sentidos heterogéneos sobre la misma en el transcurrir por la experiencia escolar y con la intervención de diferentes procesos y sujetos. En primer lugar, advertimos que estos sentidos se configuran a partir de cierto desconocimiento/confusiones sobre la modalidad y, particularmente, sobre algunos aspectos que suelen asociarse a la formación técnica. También circulan y son apropiados por los/as estudiantes, sentidos que vinculan el título técnico con la posibilidad de conseguir un "mejor" trabajo, aunque esta valoración es tensionada por otros aspectos, tales como la edad aproximada con la que finalizarían la escuela. Respecto a los conocimientos específicos que supone la orientación en administración y gestión, vimos que algunos/as los consideran relevantes para sus trabajos actuales o deseados, mientras otros/as no encuentran una relación directa entre dichos conocimientos y las inserciones laborales.

El trabajo y las tareas domésticas y de cuidado de niños/as son destacadas por los/as jóvenes como obstaculizadoras para el sostenimiento de la escolaridad. Más allá de las facilidades brindadas por la escuela, éstas resultan insuficientes frente al desgaste físico y el cansancio que conllevan tales responsabilidades, pudiendo influir en el desarrollo de los aprendizajes.

Si bien los sentidos que construyen los y las jóvenes son heterogéneos, en términos generales están atravesados fuertemente por el vínculo que se establece entre la educación y el trabajo, entendiendo a la escolaridad como posibilitadora del acceso a mejores condiciones de vida. Ahora bien, para acercarnos a comprender el modo en que se teje la relación entre educación y trabajo, fue necesario considerar los múltiples condicionamientos que atraviesan la construcción de las experiencias de los y las jóvenes, tales como las condiciones de vida de éstos/as y sus familias, sus experiencias escolares previas, sus experiencias laborales, aspectos institucionales y de las políticas educativas que imprimen particularidades a la vida cotidiana escolar, así como las múltiples relaciones que se establecen entre los distintos sujetos que participan de la misma. 
REVISTA DE LA ESCUELA DE CIENCIAS DE LA EdUCACIÓN, AÑO 17, NRO. 16, VOL. 2, JULIO A DICIEMBRE DE 2021. PÁGINAS 47-56. ISSN 2362-3349 (EN LíNEA). JÓVENES, ESCUELA TÉCNICA Y TRABAJO EXPERIENCIAS DE ESCOLARIZACIÓN EN CONTEXTOS DE POBREZA URBANA (ROSARIO, SANTA FE). FLORENCIA DEBONIS. MARILÍN LÓPEZ FITTIPALDI. MERCEDES SACCONE.

Referencias bibliográficas

Achilli, E. (1999). Antropología y Educación. Algunas claves para pensar el sentido de un programa de trabajo. En: Bernardi, G. y Nemcovsky, M. (2002). Antropología y Educación. Re-conociendo la cotidianeidad escolar. Rosario: CeaCu, FHyA, UNR.

Achilli, E. (2005). Investigar en Antropología Social. Los desafíos de transmitir un oficio. Rosario, Argentina: Laborde.

Almandoz, M. R. (2010). Políticas para la educación técnico-profesional en la Argentina. Educación y trabajo: articulaciones y políticas, 233-274. Buenos Aires: IIEP-UNESCO.

Álvarez, G. (2013). Encuesta Nacional de Trayectoria de Egresados 2013. Buenos Aires: Ministerio de Educación de la Nación - Instituto Nacional de Educación Tecnológica. http://www.inet.edu.ar/wpcontent/uploads/2015/12/ENTE-2013-Resultados-Definitivos.pdf

Binstock, G. y Cerrutti, M. (2017). Escuelas técnicas. Características institucionales y desempeños. Buenos Aires: CENEP-Ministerio de Educación-Secretaría de Evaluación Educativa. https://www.argentina.gob.ar/sites/default/files/escuelas_tenicas_caracteristicas_institucionales_y_desempen os web a4 simple.pdf

Bloj, C. (2017). Trayectorias de mujeres. Educación técnico-profesional y trabajo en la Argentina. Santiago de Chile: CEPAL. http://repositorio.cepal.org/bitstream/handle/11362/41230/S1700218 es.pdf?sequence=1\&isAllowed =y

Bourdieu, P. (2002). La juventud no es más que una palabra. En: Bourdieu, P. (Ed.) Sociología y cultura, 63-173. México: Editorial Grijalbo.

Bourdieu, P. y Passeron, J-C. (1996 [original 1979]). La reproducción. Elementos para una teoría del sistema de enseñanza. México: Fontamarra.

Butti, F. y Florez, M. (2018). Los diversos sentidos acerca de la formación para el trabajo. El caso de una Escuela Técnica Nocturna del interior del Chaco. Revista de Educación, IX (15), 169-181. https://fh.mdp.edu.ar/revistas/index.php/r_educ/article/view/2938/3243

Cerletti, L. (2009). Tensiones y sentidos de la educación infantil para los adultos involucrados. Aportes desde la etnografía. Revista Educación, Lenguaje y Sociedad, VI (6), 291-314. http://www.biblioteca.unlpam.edu.ar/pubpdf/ieles/n06a-15cerletti.pdf

Corica, A. (2012). Las expectativas sobre el futuro educativo y laboral de jóvenes de la escuela secundaria: entre lo posible y lo deseable. Última Década, (36), 71-95. http://www.scielo.cl/pdf/udecada/v20n36/art04.pdf

Cruz, Y.; et al. (2019). Preguntas acerca de las trayectorias educativas de los/as jóvenes de una escuela de educación técnica de El Carril. En Robin, S. (Ed.), Libro de resúmenes. Congreso Internacional de Educación y Política en el camino hacia un Nuevo Humanismo, 199-202. Tucumán: FFyL, UNT. http://filo.unt.edu.ar/congresoeducacion-y-politica/

Debonis, F.; López Fittipaldi, M. y Saccone, M. (2018). Educación especial, de gestión social y técnico-profesional en el Proyecto de Ley de Educación provincial (Santa Fe, Argentina). En Abate Daga, M. y Rodríguez Rocha, E. (Eds.), Tensiones entre lo público, lo privado y lo estatal en tramas educativas situadas: IV Seminario Taller Red de Antropología y Educación, 213-221. https://rdu.unc.edu.ar/handle/11086/11757

Dussel, I. y Pineau, P. (1995). De cuando la clase obrera entró al paraíso: la educación técnica estatal en el primer peronismo. En: A. Puiggrós (Dir.), Historia de la educación en la Argentina. Tomo VI. Discursos pedagógicos e imaginario social en el peronismo (1945-1955), 107-176. Buenos Aires: Editorial Galerna.

Ezpeleta, J. y Rockwell, E. (1985). La escuela: relato de un proceso de construcción inconcluso. Educação na América Latina, Os modelos teóricos e realidade social, 151-172. São Paulo: Cortez Editora-Editora Autores Asociados.

Filmus, D.; Miranda, A. y Zelarayan, J. (2001). En el mercado de trabajo, ¿el saber no ocupa lugar?: egresados de la escuela media y primer año de inserción laboral. 5to Congreso Nacional de Estudios del Trabajo, 1-25. https://www.aset.org.ar/congresos/5/PDF/FilmusMiranda.PDF

Foglino, A.; Falconi, O. y López, E. (2008). Una aproximación a la construcción de la experiencia escolar de adolescentes y jóvenes de grupos sociales urbanos en condiciones de pobreza en Córdoba. Cuadernos de Educación, 6(6), pp.227-243. http://www.revistas.unc.edu.ar/index.php/Cuadernos/article/viewFile/755/712

Gallart, M. A. (2006). La escuela técnica industrial en Argentina. ¿Un modelo para armar? Montevideo: Cinterfor-OIT. http://www.oitcinterfor.org/sites/default/files/file_publicacion/gallart1.pdf

García, J. (2013). Enfoque etnográfico y "bachilleratos populares": negociaciones entre el tiempo escolar y la autogestión de los aprendizajes. Jornadas de Sociología. Facultad de Ciencias Sociales. Buenos Aires: UBA. http://www.aacademica.org/000-038/613

Gramsci, A. (2004). Antología [Traducción de Manuel Sacristán]. Buenos Aires: Siglo XXI.

Guzmán, C. y Saucedo, C. (Eds.) (2007). La voz de los estudiantes. Experiencias en torno a la escuela. México: UNAM/Ediciones Pomares.

Heller, A. (1987). Sociología de la vida cotidiana. Barcelona: Península.

Instituto de Gestión de Ciudades. (2010). Situación de las infancias en el Distrito Oeste de la ciudad de Rosario. Diagnóstico participativo. Rosario: Instituto de Gestión de las Ciudades-Municipalidad de Rosario. http://www.igc.org.ar/Situacion-de-las-infancias-Distrito-Oeste-Rosario.pdf

Jacinto, C. (2018). El secundario vale. Saberes, certificados y títulos técnicos en la inserción laboral de jóvenes. Buenos Aires: Miño y Dávila.

Kessler, G. (2002). La experiencia escolar fragmentada. Estudiantes y docentes en la escuela media en Buenos Aires. Buenos Aires: IIPE-UNESCO.

Kornblit, S. (2004). Representaciones sociales y valores de los jóvenes argentinos en relación con el trabajo. Ciudadanía, democracia $y$ valores en sociedades plurales, 4 https://www.oei.es/historico/valores2/monografias/monografia04/reflexion04.htm

Kosik, K. (1967). Dialéctica de lo concreto. México, D.F.: Grijalbo.

Llinás, P. (2009). Imágenes y sentidos sobre la experiencia escolar: percepciones de los estudiantes sobre la escuela secundaria. Propuesta Educativa, 32, 95-104. http://propuestaeducativa.flacso.org.ar/wpcontent/uploads/2019/12/articulo_llinas.pdf

Llomovatte, S. (1991). Adolescentes entre la escuela y el trabajo. Buenos Aires: Miño y Dávila

Maldonado, M. (2000). Una escuela dentro de otra escuela. Un enfoque antropológico sobre los estudiantes secundarios en una escuela pública de los '90. Buenos Aires: Eudeba. 
REVISTA DE LA ESCUELA DE CIENCIAS DE LA EdUCACIÓN, AÑO 17, NRO. 16, VOL. 2, JULIO A DICIEMBRE DE 2021. PÁGINAS 47-56. ISSN 2362-3349 (EN LíNEA). JÓVENES, ESCUELA TÉCNICA Y TRABAJO EXPERIENCIAS DE ESCOLARIZACIÓN EN CONTEXTOS DE POBREZA URBANA (ROSARIO, SANTA FE). FLORENCIA DEBONIS. MARILÍN LÓPEZ FITTIPALDI. MERCEDES SACCONE.

Maturo, Y. (2018). El derecho a la educación técnico profesional. Efectos de la dinámica exclusión incluyente en una escuela técnica de Córdoba. Educación, Formación e Investigación, 4 (7), 55-79. http://ppct.caicyt.gov.ar/index.php/efi/article/view/13396/45454575759312

Menéndez, E. (2010). La parte negada de la cultura. Relativismo, diferencias y racismo. Rosario: Prohistoria Ediciones.

Molina, G. (2013). Género y sexualidades entre estudiantes secundarios. Un estudio etnográfico en escuelas cordobesas. Buenos Aires: Miño y Dávila.

Montesinos, M. P.; Sinisi, L. y Schoo, S. (2009). Sentidos en torno a la "obligatoriedad" de la educación secundaria. Buenos Aires: DINIECE, Ministerio de Educación de la Nación.

Nemcovsky, M. et. al. (2020). Jóvenes y escuela secundaria. Un estudio antropológico en contextos de pobreza urbana y distintas violencias. Rosario: Laborde Libros Editor.

Otero, A. y Miranda, A. (2005). Diversidad y desigualdad en los tránsitos de los egresados de la escuela secundaria. Revista Mexicana de Investigación Educativa, X (25), 393-417. http://bibliotecavirtual.clacso.org.ar/Argentina/flacso-ar/20121206022501/no19 Otero.pd

Rockwell, E. (1987). Repensando institución: una lectura de Gramsci. Documento DIE. México: Departamento de Investigaciones Educativas, CINVESTAV, IPN.

Rockwell, E. (2009). La experiencia etnográfica. Historia y cultura en los procesos educativos. Buenos Aires: Paidós.

Rockwell, E. (2018). Vivir entre escuelas: relatos y presencias. Antología. Buenos Aires: CLACSO http://biblioteca.clacso.edu.ar/clacso/se/20180223024326/Antologia_Elsie_Rockwell.pdf

Saccone, M. (2019). Nocturnidad en la educación secundaria técnica. Análisis de una experiencia en la provincia de Santa Fe (Argentina). Revista Educación, Política y Sociedad, 4 (1), 109-123. https://revistas.uam.es/reps/article/view/12313

Saccone, M. (2020). Experiencias escolares de jóvenes en contextos de pobreza. Un estudio socio-antropológico en una escuela secundaria en la ciudad de Rosario (Santa Fe, Argentina) [Tesis doctoral no publicada]. Buenos Aires: FFyL, UBA.

Sanchez, S. (Comp.) (2005). El mundo de los jóvenes en la ciudad. Rosario: CeaCu ediciones-Laborde editor.

Seoane, V. (2013). Géneros, cuerpos y sexualidades. Experiencias de mujeres en escuelas técnicas de la ciudad de La Plata [Tesis doctoral]. Buenos Aires: https://repositorio.flacsoandes.edu.ec/handle/10469/6397

Sinisi, L.; Montesinos, M.P. y Schoo, S. (2010). Trayectorias socio-educativas de jóvenes y adultos y sus experiencias con la escuela media. Buenos Aires: Área de Investigación y Evaluación de Programas, DiNIECE. Ministerio de Educación. https://www.argentina.gob.ar/sites/default/files/01-serie-investigacion.pdf

SITEAL (2013). ¿Por qué los adolescentes dejan la escuela? Buenos Aires/Madrid: IIPEUNESCO.http://archivo.siteal.iipe.unesco.org/sites/default/files/siteal_2013_03_13_dd_28_0.pdf

Terigi, F. (2010). Las cronologías de aprendizaje: un concepto para pensar las trayectorias escolares. Jornada de Apertura Del Ciclo Lectivo. Santa Rosa, La Pampa. https://www.chubut.edu.ar/concurso/material/concursos/Terigi_Conferencia.pdf

Thompson, E.P. (2012, original 1963). La formación de la clase obrera en Inglaterra. Madrid: Editorial Capitán Swing.

Thompson, E. P. (1981). Miseria de la teoría. Barcelona: Editorial Crítica.

Tiramonti, G. (2012). Variaciones sobre la forma escolar. Límites y posibilidades de la escuela media. Rosario: Homo Sapiens Ediciones.

Weiss, E. (Coord.) (2012). Jóvenes y bachillerato. México: ANUIES.

\section{Fuentes}

Instituto Nacional de Educación Tecnológica (INET) (2017). La educación técnico profesional en cifras 2017. Informe estadístico nacional. Buenos Aires: INET-Ministerio de Educación. http://www.inet.edu.ar/wpcontent/uploads/2018/03/ETP-Cifras-2017.pdf

Ley de Educación Nacional № 26.206 (2006). http://www.mcye.gov.ar/doc_pdf/ley_de_educ_nac.pdf

Ley de Educación Técnico Profesional № 26.058 (2005). http://www.inet.edu.ar/wp-content/uploads/2012/10/ley26058.pdf

Ley Federal de Educación № 24.195 (1993). http://www.bnm.me.gov.ar/giga1/normas/4572.pdf

Poder Ejecutivo de la provincia de Santa Fe (2011). Decreto No 1446/11. https://campuseducativo.santafe.gob.ar/wpcontent/uploads/Decreto-N\%C2\%BA-1446-11-R\%C3\%A9gimen-de-Pr\%C3\%A1cticas-Profesionalizantes.pdf 